\title{
Belphégor
}

\section{Alexandre Dumas : le parler noir}

\section{Charles Grivel}

\section{(2) OpenEdition}

\section{Journals}

Electronic version

URL: https://journals.openedition.org/belphegor/1208

DOI: 10.4000/belphegor.1208

ISSN: 1499-7185

\section{Publisher}

LPCM

\section{Electronic reference}

Charles Grivel, "Alexandre Dumas : le parler noir", Belphégor [Online], 16-1 | 2018, Online since 17 July 2018, connection on 19 October 2021. URL: http://journals.openedition.org/belphegor/1208 ; DOl: https://doi.org/10.4000/belphegor.1208

This text was automatically generated on 19 October 2021.

\section{(c) (i) (9)}

Belphégor est mis à disposition selon les termes de la Licence Creative Commons Attribution - Pas d'Utilisation Commerciale - Pas de Modification 4.0 International. 


\title{
Alexandre Dumas : le parler noir
}

\author{
Charles Grivel
}

\section{REFERENCES}

«Alexandre Dumas - Le parler noir » in Trames, Littérature populaire, peuple, nation, région (Actes du colloque international des 18-19-20 mars 1986), Limoges, 1987.

1 1. La littérature - populaire, aussi bien - est une adaptation: adaptation de l'impossible, adaptation de l'improbable, adaptation de l'autre, à celui qui s'en sert, qu'il la fasse ou qu'il la lise.

2 Un noir figure au fond de nos bibliothèques, au fond de nos mémoires, à cette place inquiétante : celle du sauvage (généralement «bon »), accompagnateur du Père Noël ou serviteur agenouillé lippu des écoles du dimanche et des quêtes. A moins qu'il ne vende ses ivoires et ses sculptures sur les quais. L'autre, en somme, sous forme de la bête. Docile, encarcané, balbutiant (sans aucun « $r »$ ), etc. La littérature - celle de Dumas, pour commencer - trame ce fil-là. Je voudrais le remonter. De lui à nous.

Qu'est-ce qu'un nègre ? L'autre de couleur. Un blanc changé de couleur - Forneret a su le démontrer. Le sauvage intérieur mais vu dedans, une apparence. Comme si la pensée ne pouvait se développer que sur deux faces à la fois, celle qui est exposée - «blanche » et celle qui est cachée - obscure bien sûr. L'Africain, support de l'amour autant que du rejet. L'imaginaire connaît volontiers de ces situations-là : un fantôme est chargé d'évacuer certain trop-plein de l'âme, mais il revient, passé minuit, hanter celui qu'il oblige. Double face des noirs de nos littératures : tout méchants, directement calqués $\mathrm{du}$ diable, et tout bons, propres à venir s'étaler dans les draps des hospices et des missions - mais aussi chargés de représenter le manque d'être d'Européens exsangues, ratiocinateurs et dépourvus de muscles (au genou ou à la voix) : le coureur noir, le buteur noir, le chanteur noir, le cavalier noir, le révolutionnaire noir - de Bug-Jargal à Georges, de Hugo à Dumas, de Dumas à Yannick Noah ou à Harlem Désir.

4 J'insisterai dans l'étoffe du mythe, je fouillerai l'auteur du Monte-Cristo, semi-noir luimême, afin de faire jaillir de son texte le présupposé très renforcé - moteur d'après 
moi -, négrophile et négrophobe, qui s'y tient. Dumas: de la hantise d'une identité non-mêlée et sans tache. D'un racisme appliqué d'abord à soi-même, et de là gagnant tout, comme une peste, l'écriture et chacun de ceux que je ne suis pas. Je m'intéresserai donc, tout naturellement, aux motifs d'exclusion et d'épurement des fables, car ils sont «noirs». Et je regarderai, comme nous tous, car je vois l'homme de couleur irrésistiblement nous revenir, vers Le Cap.

5 2. Dumas donc, fils d'Alexandre (le général), petit-fils d'Alexandre (le colon), père d'Alexandre (l'homme de théâtre). Des textes - un monceau de textes ! - articulés à une filiation. Que ces textes passent pour populaires, peu importe: disons qu'ils existent, fonctionnent, plaisent. Disons qu'eux aussi sont des machines : ils produisent un mouvement sur leur propre énergie. Disons encore qu'ils brassent l'imaginaire et engendrent là, pour leur auteur et pour nous, la plus désirable des jouissances-tête. "Moi, je prends mes sujets dans mes rêves, écrit Dumas, je travaille les yeux fermés » ${ }^{1}$ : il faut prendre cette déclaration au sérieux : quelle que soit l'apparente origine du récit dumasien, c'est de source mémorielle qu'il coule; ce qu'il conte est l'histoire de sous l'histoire, un affleurement du fond secret où la main puise pour écrire. Une " inconnue " commande le récit, un $\mathrm{x}$, un «noir » - c'est mon idée -, un noir qui se blanchit - je vais le montrer -, avec et sans aucun savoir de la part de son auteur et dans une France comme par hasard "restaurée ", éternel retour et éternelle coloration, qui vire au blanc.

6 Exemplarité, à maints égards, du texte dumasien : il entraîne, il séduit, il s'applique. Il réussit son développement imaginaire, il pousse jusqu'au bout de ses propres données (ces données ne sont pas forcément celles qu'on croit), il enchaîne les scènes et dans la langue il parle. Son intérêt théorique (cet intérêt est bien plus facile à désigner que l'autre) est alors qu'il dise ce qu'il dit non seulement dans l'inconscience (mesurée) propre à tout ouvrage littéraire de fiction, mais qu'il le dise sous dénégation, dans un langage "blanc", supposé ne vouloir rien dire outre la fable et outre l'amusement qu'elle nous procure. D'un discours qui serait léger. D’un récit pur de la détente. Je vais devoir démentir : Dumas parle, à contre-jeu de ce que lui-même s'efforce de nous faire entendre, fort bien, mais il parle. Je le vois dès que je soulève les mots.

On corrigera le dit-on : Alexandre Dumas est bien l'auteur de ses livres, il est son propre nègre! Je pose en principe que tout ce qui est signé «Dumas » est - d'une certaine façon - identifiable à lui. Pourquoi ? Tout simplement parce qu'il l'a reconnu pour sien. Je ne fais pas de différence entre le tout écrit, l'un peu écrit, le pas du tout écrit ou récrit de notre auteur. Sur cette conviction que, même à court d'inventions, il ne s'est servi que de ce qui, en profondeur, lui convenait.

Du reste, ces histoires que Dumas s'est fait - comme bien d'autres - profession de raconter, d'où viennent-elles et d'où peuvent-elles venir? Le monde est un sac à histoires : il suffit de l'ouvrir et d'y plonger la main - la plume. Le monde est l'occasion d'une histoire. Tout se passe comme si, venant au monde, le sujet se trouvait précipité dans le racontement infini de ce qui se passe, pourrait ou devrait se passer. Dumas, en tout cas, a toujours été affamé d'histoires. Il vit à l'affût du récit d'autrui - non pas parce que la source en lui de raconter serait tarie, mais parce que toute histoire est son bien, lieu du propre, lieu de la reconnaissance, miroir qui ne demande qu'à être ajusté signé. Que je lise ou que je me déplace, le long des rayons de la bibliothèque ou au cours de tous mes voyages, des histoires se déroulent. Je chasse un conte ; un conte m'arrive. $\mathrm{Du}$ reste, la rencontre du voyageur et du récit ne se fait pas par hasard; bien au 
contraire, elle se prémédite ; ainsi, Dumas provoque-t-il le surgissement du réel - à Heidelberg, par exemple, le fils du bourreau qui exécuta Karl Sand - du personnage qu'un récit lui destine et qu'il compte à son tour replacer dans la fable. Considérons donc plutôt le récit comme une substance, une mine à exploiter, un « or » dont l'origine importe moins que l'applicabilité. Tout récit est monnayable; c'est cela, il me rentre dans l'esprit; je le tiens, il me lit.

9 3. Je propose ici l'étude de la compulsion d'identité du texte dumasien, de livre en livre, dans le flux même de ce qui me parait le susciter. Mon idée est celle-ci : un texte va à ses hantises (tout peut le freiner sur ce chemin, je ne dis pas), il est poussé par elles à ses limites; il s'excède, il se varie; une œuvre est cela : infini réajustement d'un dire qui se cherche, d'une parole qui s'accomplit, précipitation à une fin qui, sans doute, ne se trouvera jamais. Un mécanisme se met en place, un contenu s'articule, un fond se gagne ou émerge : du récit sourd ce qui en est la base - sa fantasmatique, son imaginaire. Ecrire ou raconter ne coïncide avec aucune histoire qu'il agence, ne se réduit jamais à son fil. Ecrire ou raconter insiste, produit, travaille : Dumas, la chose-Dumas, l'identité mémorielle Dumas est tout d'abord ce que ses multiples écrits ont, couche à couche, et pour nous, lentement constitué. Le récit exploite donc moins un donné préalable qu'il ne l'élabore lui-même au gré de son exercice. L'imaginaire que nous lisons sous trame arrive à son auteur, au bout de l'infinie figuration à laquelle il se livre. Une histoire s'ajoute à une histoire ; elle s'y substitue et s'y ajoute, formant dépôt. J'écris (je lis) dans l'épaisseur. Un processus d'appropriement identificatoire est en cours : ce que je conte, en effet, me cerne et me poursuit. Le «moi » est une scène peu à peu comble, que je remplis entassant mot sur mot, par affamement de moi-même. Sur la scène, plusieurs pistes, plusieurs lieux, plusieurs acteurs - moi toujours, moi tenté, moi accru, repris, rendu. Penser à une mosaïque de plus en plus complexe, à un puzzle de plus en plus complet, dont les pièces s'encastrent avec de plus en plus d'exactitude et de finesse. Ce que je dis de texte en texte et par histoires interposées se garnit comme un tableau; bientôt il n'y manquera plus aucune pièce ; cependant, les choses sont ainsi que son achèvement laisse toujours à désirer : pourquoi ?

10 J'ai beaucoup lu Dumas. C'est l'impression que j'en retire : que les pièces s'imbriquent, se complètent, qu'il en manquait nécessairement une, que voici, qu'un dessein se poursuit avec opiniâtreté, progressivement, ajustant, accommodant, complétant toujours avec inattendu d'un morceau l'édifice mémoriel qu'on aurait dit terminé, à chaque fois plus vrai, je pense.

11 Au centre de cette mosaïque mentale que constitue l'œuvre, une pièce maîtresse, une figure, celle du noir. Je dis « figure » : c'est à la fois un personnage et une couleur, une idée et une chose, un signe et le nom que porte l'irreprésentable, l'autre, l'étranger par excellence, la marque impure, que je dis que je n'ai pas, dont je me débarrasse : le noir, symbole des différences; symbole des identités aussi : il est celui par quoi je me pense (je m'accepte et je me pense, corps compris), l'antithèse d'où je tire ma très désirable blancheur, celui qui m'épure et me lave. Voilà ce que je crois qu'il faut que j'exhume.

4. Qu'est-ce que le noir pour Dumas? Il est une façon simple de répondre et une autre, sous-jacente, qui nous préoccupera. Pour commencer, comme chacun sait, Dumas est fils de bâtard reconnu tardivement et quarteron, sa grand'mère paternelle ayant été esclave à Saint-Domingue. "Dumas » est, du reste, le patronyme de celle-ci : un signe " nègre » sous le nom du père (puisque celui-ci l'avait adopté également) vient marquer d'un indice ambigu les écrits de l'auteur (Dumas réservant le nom de son grand-père 
auquel il a droit aux pièces officielles). Par ailleurs, chose notoire aussi, Dumas possède la morphologie du noir (il est lippu, crépu), son caractère (c'est un " cœur d'or », un «naif » - je reproduis le diagnostic) et ses goûts (Nodier à Dumas grand amateur de bijoux: "Ah! vous serez toujours les mêmes, vous autres nègres... Vous aimez les verroteries et les hochets $»^{2}$ ). Ce n'est pas tout. Par un curieux paradoxe qui n'avait pas échappé aux contemporains, Dumas, nègre lui-même, emploie des «nègres » à ses écrits : "Grattez l'œuvre de M. Dumas, et vous trouverez le sauvage (...). Il embauche des transfuges de l'intelligence, des traducteurs à gages qui se ravalent à la condition de nègres travaillant sous le fouet d'un mulâtre $»^{3}$. Ceci n'est que la façade. Je vais montrer un peu de tout ce qui se joue en contre-champ, par écrit, de ce destin-là. L'œuvre de Dumas est une entreprise d'épuration, un blanchissement secret, rentré, infiniment complexe et contourné. Comment se dépourvoir, en effet, de cette « couleur »- de ce signe - qui nous altère et pourtant nous différencie, valorise, sans désaveu ni déni ? Toute une gymnastique narrative va être mise en place pour cela.

Reprenons d'un peu haut, en plein dans les fantasmes de l'Occident, puisque aussi bien tout l'imaginaire dumasien y trouve sa source. Du nègre et du blanc, comme d'une opposition motrice à partir de laquelle le devenir trouve à se penser - la nature, la personne. Le premier figure aux deux bouts de la chaîne évolutive ou divine, cela dépend si l'on suit le schéma christique ou le schéma positiviste: le blanc est au commencement - Dieu s'y est mis, Dieu s'y est conçu -, le blanc est à la fin - la nature, après tous ses errements, y retourne ${ }^{4}-$. De toutes façons, le blanc provient et revient à l'être ; il en est l'authentique réplique : Dieu ne se voit que blanc. Inversement - car dans cette logique ne figurent que deux teintes -, le noir est antérieur ou postérieur; il est au fond, comme le signe d'un chaos que l'acte de création nie, il surgit, faisant tache, comme l'indélébile marque des créatures d'après la chute. Indétermination ou bien déviance. Le blanc seul est originaire ( Le blanc est la couleur des premiers hommes $\left.»^{5}\right)$. Une tache de couleur advient sur ce blanc dont la pureté, par définition, est en passe d'être salie. Blanc salissant, noir dégradant: comment ôter la tâche? Comment l'ôter surtout si l'on est né soi-même du mauvais côté du symbole détestablement noir?

Tous Dumas ; tous un nègre dans la gorge, comme ancêtre et comme refoulé. La nuit au fond de soi, l'inqualifiable, la souillure : d'où ces récits qui nous conviennent, d'où ces luttes qui nous affectent. Dumas : nous nous trouvons au centre imaginaire : d'où je crois que provient ce que je ne suis pas - d'où me vient l'autre - mon bâtard - et mon diable.

15 Car le nègre est dessous ; il est caché ; il se confond avec la mémoire ; il est le désordre, la crise, l'intérieur et primordial chaos, le sauvage primitif et le primate qui revient. Intervention implacable du désordre originel, immanquable salissure d'une génération qui ne tient pas, le noir remonte et ressurgit. Comme un double négatif toujours près de franchir la ligne des apparences. Une phase inquiète domine ainsi la bonne : me voici blanc avec un nègre dedans moi, " métissé », « mulâtré », tel Dumas. Un " trou » mine mon discours (le fruit avec un ver, le paradis avec un diable). Une catastrophe que je crois génétique creuse un puits - un principe d'étouffement - au fond de moi : mon imaginaire creuse mes textes - me les «noircit ». Mes textes exhument mon propre, me le bouchent et me le transforment. Un noir écrit mes textes. Je ruine et fouille le blanc ; je trouve et ruine Je nègre. Je me nettoie après souillure - avec imaginaire - avec littérature - je me suis refait le baptême. 

qu'écrire et lire brassent, aménagent, distribuent et très sciemment appliquent. Vous parlez toujours d'une base identificatoire. Vous êtes le bon ou le méchant (à la fois, successivement) de cette base, identité plus identité, identité faisant défaut, identité à perte : "Quand on est de la couleur qu'il faut, on ne se fait jamais prendre, murmura-til dans un souffle, mais les deux autres l'entendirent» - interjecte McAfee (sic), le contrebassiste noir d'un récit de Pynchon ${ }^{6}$. Au blanc, la transparence, l'impunité ; au noir, l'épaisseur, la balourdise, la faute. Ce principe économique vous permet de situer votre personnage et de le signifier outre et hors conscience : blanchissez-le sous une ombrelle ou passez-le au cirage, ce que vous ferez paraitre dans le teint sera compris sans que vous vous en mêliez dans le bon sens. Une partition symbolique constitue le récit dumasien : des énoncés vous arrivent scindés dans une histoire. Vous ne le voyez pas bien, pas forcément, ils vous retournent la pensée sans que vous vous y attendiez. Vous avez lu. Quelque chose a changé de place. A été transmuté. Bonifié, mais cependant expulsé. Quoi ? L'être noir, précisément, l'inadmissible père truqué.

Du reste, la question nous propulse au centre même de l'opération d'écriture. Il vaut la peine de s'en rendre compte: les noirs ne hantent pas seulement l'Afrique, ils encombrent le livre et leur couleur-peau est, pour commencer, typographique. Blanc/ noir vient au bout de la plume; c'est le signe qui ressort d'une encre dessinée sur la page. Je trace dans le double registre ; je pense par le biais de marques que ces deux « couleurs »- exclusives et sans couleur - composent. Le contraste me sert : il me fait voir. Je ne distingue que sur le fond les objets découpés que ma rétine et ma main cernent: le cerne est noir, la marge est blanche. La différence seule fait sens et la différence ne s'obtient que par couplage/découplage des concepts - par le jeu des contraires et des oppositions - par le choc qu'ils procurent. Il me faut un, deux et puis la division, noir/blanc, par couple, dans le couple, amour d'un côté, rupture de l'autre. La clarification de mes données parait être à ce prix. Une page, un livre, écrire, font la différence. Je raconte en opposant du noir au blanc - par les signes que j'arrange, selon les personnages que j'engendre, un bon, un méchant, l'un coiffé de noir, l'autre peigné de blond : un bon conteur - Dumas tout le premier - n'en finit pas de soigner ces apparences-là.

Blancheur du papier, noirceur de la lettre, cet antagonisme figure à l'origine du discours que je tiens, ces deux matières ou ces deux fonds se faisant pendant dans l'inertie à être dits : le blanc est un barrage, le noir est un barrage aussi. Cela est trop rempli de mots - comment faire surgir de la rumeur une parole propre ? Cela est trop vide de mots - comment faire surgir du non-sens un sens vrai ? $^{7}$ «L'angoisse de la page blanche existe (...), qui retient d'écrire et concentre sur la première phrase la douleur de commencer " ${ }^{8}$. Et Mallarmé : "L'effroi d'une feuille de papier blanc, qui semble demander les vers si longtemps rêvés (...) qui a fini tellement par faire partie de vousmême qu'on l'a oubliée, comme le reste de soi $»^{9}$. Dumas en quête du récit - ne résistons pas aux comparaisons sacrilèges! - paraît, lui, noircir le papier, feuille à feuille encombrer les volumes, à propos d'un sens qui ne vient pas, comme repoussé devant à mesure que la plume se déplace et trempe dans l'encre l'imaginaire requis. Vertige de la page blanche, vertige de celle qui se noircit : comme si l'anxiété d'avoir à rencontrer de quoi parler renforçait la langue dans la bouche ou alimentait désespérément sa faconde - de la même façon. Loquacité - silence, même arrêt devant 
ce qui ne veut pas sortir, même inertie : le vers à vers du poème, le vague à vague du récit laissent intact, à leur façon, ce qu'ils manquent à révéler.

Du blanc est cela que j'écris, effacement des rumeurs, oblitération du noir, du bruit, du parasitage, du sens, trop nombreux et trop inadéquat. Du blanc serait écrit. Du blanc me fait écrire. Poursuite vaine d'une parole sans tache, "poétique ", enfin advenue. Mallarmé dit: «Les blancs assument l'importance». Ratage: "Le blanc est insaisissable ». Il figure cet espace creux du livre, où celui-ci perd son propos : «Le blanc, l'espacement lui- même, la différence, n'est ni ici ni ailleurs, pas saisissable dans un ici maintenant mais toujours plus loin, autre part. Elle n'est pas quelque chose $»^{10}$. Blanc, figure de la contradiction : ce que j'ai à dire provient de et se détache sur le fond d'une langue qui n'est pas mienne. Je cède à cette langue, elle m'occupe ; mais si je n'y cède pas, le silence forcé où je me tiens tout autant en provient. Double traquage que la demande d'un parler propre entraîne. Dumas compulse le noir: des heures et des heures d'écriture de récit chaque jour, voué lui aussi dans la contradiction à l'extermination de ce qui précisément lui fait prendre la plume : l'obscurité chaotique et le nègre antérieur. Un signe est en trop ; j'ai le malheur qu'il m'est apparu - inscrit, pis, dans ma chair : je n'aurai de cesse que je l'en aurai radié. Mallarmé encore, à propos de l'opposition fondatrice: "Tout devient suspens, disposition fragmentaire avec alternance et vis-à-vis, concourant au rythme total, lequel serait le poème tu, aux blancs ; seulement traduit, en une manière, par chaque pendentif ${ }^{11}$. On peut tenter de figurer l'intérêt qui vient de cette lutte, entre un noir rebelle et la blancheur divine: c'est ce que Dumas fait. Cet intérêt échappe au texte. Cet intérêt est nié par fiction. Mallarmé enfin : «L'encrier, cristal comme une conscience, avec sa goutte, au fond, de ténèbres relative à ce que quelque chose soit: puis écarte la lampe (...) L'homme poursuit noir sur blanc »- inversement donc au langage des dieux, céleste, à base noire, tout de chaos, primordial et enfin rendu à sa fatalité silencieuse ${ }^{12}$. Double inverse des opérations : de l'homme à son ciel, de son écriture aux étoiles, de sa noirceur à sa blancheur. Dumas : une identité se gagnerait au croisement des deux notions. Les récits seraient des sas d'épuration - d'impossibles et nécessaires stations. "La tache d'encre est bien originelle (...) La tâche est toujours grosse, pleine, dense (...) gravide »" ${ }^{13}$. C'est de cette tache - bâtardise pour mulâtrerie -, de cette «impureté » que nait le livre. Je récite dans l'horreur de cet indécidable premier. Les premières choses sont noires - elles sont ce qu'elles ne devraient pas être : elles naissent avec moi, ma présence est aussi leur intrusion, je n'aurai de cesse qu'elles ne se dissolvent. Les premières choses sont impures : vivre est accéder au noir. Bachelard : « Le noir est comme nourri de boue ${ }^{14}$. Je n'aurai de cesse de m'en discréminer.

20 5. Service nègre

21 Je réduis et centre mon propos. Je ne vais pas me hasarder à faire, via Dumas - encore que cela ne serait pas dépourvu d'intérêt -, le relevé des racismes de nos âmes, pires et bien plus ancrés que celui qui se reconnaît à l'épiderme. Bien entendu, philosophie et politique, opinion courante et idéologie pensent "nègre " aussi sur les bases que j'ai dites. Je ne chasse pas ici le nègre en société, mais m'exerce à simplement déconstruire l'armature symbolique de soutien du service qu'il remplit en régime de fiction.

Ce qui est particulier. On peut demander à quoi sert la race dans l'édifice épistémique d'une société ; je cherche seulement où va, pour quelle obscure besogne, un roman dont je perçois qu'il achoppe là : le traitement du noir, non sa traite m'intéresse. 

déplace dans les combles inapparents de l'imaginaire. Je sais, bien sûr, que la chose et la notion viennent occuper, au cours des âges, toute une série de positions variées dont je ne puis pas ne pas tenir compte. Je n'accrocherai pourtant mon étude à aucune histoire de l'imaginaire : celle-ci fait défaut et une écriture n'en accomplit jamais - loin de là le fidèle exposé. Nous ne nous trouvons pas, malgré les apparences peut-être, dans la mouvance archétypale. Quelques repères, néanmoins. Je rappelle, par exemple et sans vouloir remonter dans la nuit des temps, que notre Eglise associe noir à diable et les maures, à titre d'hérétiques, à la couleur des ténèbres. Sur cette base, le noir, toujours esclave et domestique, s'entend, avec une belle nécessité, sous l'obédience du mal. Il faudra attendre la Révolution française pour qu'on reconnaisse - sur le papier, du moins - une "raison ", et donc l' "égalité », à celui qui n'a pas la peau blanche. Les associations négrophiles et anti-esclavagistes d'alors, malgré leurs bonnes intentions, perpétuent sous couvert les idées dont elles combattent certains effets. C'est sur la base de la perturbation profonde provoquée en France par la reconnaissance officielle, mais non réelle ${ }^{15}$, de l'identité noire, que Dumas rédige ses ouvrages. L'auteur de Georges (1843), de La Tulipe noire (1850) et du Meneur de loups (1857) - puisque ces livres serviront tout particulièrement à étayer ma démonstration -, pensé comme nègre et se pensant lui aussi comme tel, écrit pour se «blanchir». L'époque n'offre aucune échappatoire: la négritude n'est pas recevable; elle ne peut servir au fondement de personne; un nègre peut être un nègre, il ne saurait posséder la propriété (avoir et être) que le blanc signe. La discrémination est totale, les identités ne commutent pas, je suis blanc de ce que tu es noir. Dumas - je le dis maintenant par hypothèse - s'acharnera à retourner la symbolique en cours pour détruire la servitude (mentale et sociale) qu'elle signifie. Ceci, par fiction interposée. En plein dans le fantasme. De l'emprisonnement de l'imaginaire dans certaines de ses représentations-clefs, de la négativité de ces représentations, de l'insupportabilité de ces représentations. J'instruis cette affaire-là : comment écrire pousse contre les limites de la pensée qui lui est donnée ? Comment modifier « dans le roman » ce que l'on est pourtant « au réel »? Je prends Dumas comme un cas exemplaire. Celui d'un esprit que le dénigrement $-\mathrm{si}$ j'ose dire! - et l'acceptation du noir, c'est-à-dire de l'impensé de sa pensée successivement et impossiblement saisit. Dumas ne peut s'imaginer d'être que nié. Lui dans son ancêtre, il lui faudra "tuer le père ". Ce père étant mort pour ainsi dire à sa naissance ${ }^{16}$, et de mort en martyr, cela ne sera pas facile.

6. Je reconstitue donc un paysage mental, je dresse la carte de l'imaginaire noir dumasien, à grands traits, on ne va jamais assez dans les détails. Cette carte est mouvante, ses éléments se déplacent, elle ne décrit pas, mais s'implique : nous avons affaire avec les trajectoires complexes et retorses - il faut mettre un pluriel - d'un discours qui bute à des limites qu'il ne comprend pas toutes, mais auxquelles il revient avec acharnement. Aucune archétypie ici - un texte n'est pas un catalogue, mais un investissement discursif à saisir sur le vif. Je procéderai en deux temps, commodité oblige : d'abord, un inventaire de ce que "noir » comprend afin de le mettre en œuvre, ensuite, quelques exemples un peu fouillés d'implication dialectique impossible dans trois cas de récits que je juge insolubles.

I. Le noir est la mauvaise couleur. Cette couleur ne s'avoue pas; elle ne se porte, ni ne se supporte ${ }^{17}$. C'est la couleur morbide par excellence, celle des maladies graves, des crises et des étouffements (BLA, II, 143, 145) ${ }^{18}$. Si je meurs, je deviens « noir »; ma vie s'en va, 
mon visage «noircit». Thérèse, jeune fille séduite, a la peau obscurcie par le choléra. Cette maladie fait d'elle ce que font des humains les plus sinistres affections - je cite « la fièvre jaune, la peste noire et le vomito-negro » (BLA, II, 152) : elle plombe la face, elle lui gomme l'apparence. La mort vient d'ailleurs; elle rend au pire, sous l'empire d'une inimaginable (dé)coloration. Le visage du mort est noir, bien évidemment, " comme de l'encre", comme ce Louis XIV qu'on ressort de son tombeau - aucune majesté ne tient! (HMR, 49). La vie se retire, par empoisonnement ou asphyxie: je deviens noir (MHP, II, 118). Je nais : je deviens noir aussi - témoin le récit que fait Dumas de sa naissance dans ses Mémoires ${ }^{19}$. De proche en proche, toute négativité participe du noir : paraît le séducteur, son nom déjà le désigne déjà à notre vindicte : «Gratien d'Elbène »! (BLA, II, 207) - et qu'il comporte en soi par métonymie celui de l'Empereur ${ }^{20}$ ne fait que renforcer la puissance néfaste qu'il accueille. Un "signe noir ", au reste, suffit à négativer la personne - ou à en sceller la perversité. Témoin le portrait de Mlle Danglars, dans Le Comte de Monte-Cristo (CMC, I, 772-773): "Mlle Danglars était belle, mais comme l'avait dit Albert, d'une beauté un peu arrêtée : ses cheveux étaient d'un beau noir, mais dans leurs ondes naturelles on remarquait une certaine rébellion à la main qui voulait leur imposer sa volonté; ses yeux, noirs comme ses cheveux, encadrés sous de magnifiques sourcils qui n'avaient qu'un défaut, celui de se froncer quelquefois, étaient surtout remarquables par une expression de fermeté qu'on était étonné de trouver dans le regard d'une femme; son nez avait les proportions exactes qu'un statuaire eût données à celui de Junon ; sa bouche seule était trop grande, mais garnie de belles dents que faisaient ressortir encore des lèvres dont le carmin trop vif tranchait avec la pâleur de son teint ; enfin un signe noir (je souligne) placé au coin de la bouche, et plus large que ne le sont d'ordinaire ces sortes de caprice de la nature, achevait de donner à cette physionomie ce caractère décidé qui effrayait quelque peu Morcerf ». Noir, couleur des corruptions, des rébellions, couleur du diable (BAM, I, 59). Triple livrée macabre de Satan - c'est ce que dit le récit $(\mathrm{FCV}, 383)$ : sa voiture est noire, ses chevaux, son cocher, son " groom » aussi, «noir d'habits et de peau ». Noirceur des conspirateurs, faux « charbonniers ", faux " nègres » - je cite le texte (SAL, I, 252) - et vrais "carbonari », zélateurs du bonapartisme dans la France des années Louis-Philippardes. Au reste, les conjurés se rencontrent sous Paris non seulement dans l'obscurité propice des catacombes, mais encore "rue d'Enfer » (SAL, 1,253). Noir, produit de l'ombre - van Baerle y trempe ses tulipes -, émanation de la greffe et du mixte - c'est par déviance et recoupement qu'il obtient la fleur miraculeuse (TUN, 69) -, inadmissible impureté faisant menace.

II. Le noir est celui d'une autre langue. Balbutiant, baragouineur, le nègre ne parle pas, il manque de " $\mathrm{r}$ » et de syntaxe pour cela. "Moi pas croire vous", dit le ToussaintLouverture de service (SAL, II, 18) - et les deux domestiques de couleur de la maison Dumas, Alexis et Paul, ne jouissent pas d'un langage plus châtié (cf. HMB, 116 sq., 249 sq.) Le noir parle noir, c'est-à-dire hors la langue, un français dépourvu de français. Infantilisation-type, que flanquent bien d'autres procédés analogues, et sur laquelle, du reste, Dumas n'insiste pas particulièrement : n'était-il pas lui-même plus que sensibilisé aux reproches qu'on lui faisait sur son style, trop "parlé », trop «coulant", trop " lâché " pour être de bonne origine? Quant à Georges, retourné parfait gentleman (et richissime) dans son île, s'il s'exprime comme vous et moi, mieux même, n'est-ce pas qu'il a réussi - nous verrons comment - sa transmutation? défavorable de la série lui convient aussi. Ainsi, le noir appelle-t-il, comme par 
contiguïté, le roux, pigment qui lui est pourtant le plus antithétique. C'est que l'un et l'autre se pensent dans la négativité, comme produits de l'impur et des mélanges. Une obscuration, une " déchéance ", une « bâtardise » figure imaginairement à l'origine des deux colorations. On conçoit dès lors ce que donne leur conjonction! Le Malin mêle le roux au noir ; la Bête, la rousseur à la noirceur. Le poil et le pigment, la " robe ", dénote tout ce que vous ne voulez pas. Association nécessaire du roux et du noir, dans cette «logique ", pour exprimer l'insistance dans le mal, l'horreur aussi des commutations, des mixtes. Ainsi perçoit-on, comme naturellement, au fil du récit, qu'un tel alliage - et tout alliage, en fait, sauf celui que la blancheur commande - fasse entendre à demimot, par exemple l'insupportabilité d'Edouard, l'enfant-démon: "Il était petit, grêle, blanc de peau comme les enfants roux, et cependant une forêt de cheveux noirs, rebelles à toute frisure, couvrait son front bombé, et, tombant sur ses épaules en encadrant son visage, redoublait la vivacité de ses yeux pleins de malice sournoise et de juvénile méchanceté » (CMC, I, 698). Le mixte, qui comprend la surcharge du noir par le roux, les deux néfastes de la langue, avec contraste à un blanc morbide qui n'est là que pour en faire ressortir la contradiction, constitue le pire signal de la pire nature. Autre exemple, avec adjonction d'un incompatible blond angélique, ce portrait d'AndreaBenedetto, en provenance lui aussi du Comte de Monte-Cristo (CMC, I, 813-814) : « C'était bien ce grand jeune homme aux courts cheveux blonds, à la barbe rousse, aux yeux noirs, dont le teint vermeil et la peau éblouissante de blancheur lui avaient été signalés par son maitre ». Noir et roux sur blanc plus blond, telle est la fiche signalétique du "mauvais ange " - je cite, car Dumas aime à mettre les points sur les i. Tout se passe donc comme si le noir, figure-type de l'hybride, nécessitait pourtant, par on ne sait quelle raison profonde, d'être resignifié par surcharge pour bien marquer ce qu'il veut dire.

IV. Le noir fait tache. Le noir ne s'efface pas. En lui joue un principe d'indélébilité, de perdurabilité. La tache noire est complète : elle gâte tout l'ouvrage. Elle est néfaste : elle implique une fâcheuse orientation du cours des choses, de la nature des êtres. Elle rappelle à l'homme, cette "tache sombre", dit Dumas (FCV, 310), que le bonheur intégral et définitif n'existe pas. Une face sombre, ne serait-ce que par son insertion microscopique au corps (ou à ce qui rejoint le corps), connote ou surimprime l'événement. Noire tacheture est ainsi la forme prise par la fatalité; son sceau s'imprime en nous, matériellement, de cette façon. Mariette écrit à Conscience, le fiancé qui a tiré le mauvais numéro ; elle fait une tache d'encre sur sa feuille; elle tente d'enlever la tâche avec la langue, mais celle-ci devient grise et - dit le texte - quatre fois plus grande après léchage (DED, II, 91-92). Pendant ce temps, au front, Conscience perd la vue : l'explosion d'un caisson d'explosifs vient confirmer le signe de mauvais augure. Inversément - mais rare apparition -, la tache blanche est bénéfique, bien sûr ; elle coule, elle se goûte, elle nourrit. Ainsi, à propos - comme à propos - d'un simple nuage « voyageant si lentement dans les plaines de l'air, qu'il semblait une tache de lait au bleu firmament » (DED, III, 71), est-ce tout l'envers immaculé du monde qui fait signe.

V. Le blanc est la bonne couleur, celle des biches et des colombes, des victimes expiatoires (DMF, II, 118-119, 130 ; TDE, 121), de ces bêtes qu'on ne chasse pas sans un sursaut de conscience (Lettre de Dumas à Van Loo, 9 octobre 1858) ${ }^{21}$. La divinité est du côté du blanc; un abondant faisceau de connotations se greffe là, symbolisation positive généralisée de ce qui se colore ou décolore ainsi : la rose - elle indique alors l'innocence (MHP, II, 66-67) -, la chambre mortuaire - elle signifie alors la virginité (MHP, II, 102). La vie 
possède, selon ce découpage, un côté pile, noir et menaçant, et un côté face, blanc " radieux ». Bonheur d'être sur le bon versant, exposé à la lumière « blanche » que Dieu commande (FM, 382). Dans la même ligne, « être voué au blanc ", c'est être voué à Dieu. Récit de cet usage in MUF, 133-134, où il est spécifié qu'il s'agit du vœu d'une mère qu'endeuille la mort successive des enfants qu'elle met au monde. Or, cette fois-ci, l'enfant vit. Blancheur. Juste après le décès du père. Moralité : le père mort, d'où vient le noir, l'enfant blanc peut paraître, sa divinité est à ce prix. Bien entendu, la scène de fiction se déroule là même où le petit Alexandre vit le jour : "Mon père et ma mère habitaient un petit village situé au milieu de la forêt de Villers-Cotterêts et qu'on appelle Fleury. Avant ma naissance, mes parents avaient déjà eu cinq enfants, trois garçons et deux filles, qui, tous, étaient morts. Il en résulta que, lorsque ma mère se vit enceinte de moi, elle me voua au blanc jusqu'à l'âge de sept ans, et mon père promit un pèlerinage à Notre-Dame de Liesse (...). Malheureusement, mon père mourut pendant la grossesse de ma mère : mais ma mère, qui était une femme pieuse, ne résolut pas moins d'accomplir le double vœu dans toute sa rigueur ; aussitôt ma naissance, je fus habillé de blanc des pieds à la tête, et, aussitôt qu'elle put marcher, ma mère entreprit à pied, comme il avait été voué, le pélerinage sacré ». Le texte dit ainsi que la bonne couleur se mérite ; elle n'échoit qu'avec la souffrance - seul quatorze ans d'emprisonnement pâlissent à point le prisonnier (CMC, I, 396, 399) 22 -, qu'en échange de la mort ou même «après la mort ». C'est le cas du héros de La Tulipe noire: Van Baerle est mené au supplice, il attend la tête sur le billot «l'horrible avalanche » (sic). Dumas écrit : «Van Baerle dit adieu à la grande tulipe noire, certain de se réveiller en disant bonjour à Dieu dans un monde fait d'une autre lumière et d'une autre couleur" (TUN, 125). Mort et transfiguration, échange de pigments. C'est que le Seigneur l'avait «regardé avec le sourire d'un père ». Peut-on dire plus dans le genre?

31 VI. Le blanc est une inconcevable, innommable couleur. Un impossible, un lointain, une inatteignable limite, que le héros, par extraordinaire, servi par un destin qui n'est qu'à lui, endosse, est chargé de nous représenter : nous souffrons de ce manque qu'il affiche, lui, vaincu dans sa peau. Portrait de Salvator : «Mais ce qu'il y avait surtout de frappant dans ce jeune homme, c'était le ton blanc, c'était la mateur de sa peau; ce ton n'était, en effet, ni la pâleur jaunâtre du savant, ni la pâleur blanche du débauché, ni la pâleur livide du criminel : pour donner une idée de la blancheur immaculée de ce visage, nous ne trouverons d'image et de comparaison que dans la pâleur mélancolique (sic) et lumineuse de la lune, dans les pétales transparentes du lotus blanc, dans la neige intacte qui couronne le front de l'Himalaya » (MHP, I, 43). Pour le reste, en vertu d'un principe que nous allons exposer, Salvator se trouve habillé et chapeauté de velours de soie noir.

VII. Le blanc n'est bien blanc que par contraste. Il n'est signifiant, intéressant, "beau ", " séduisant », que par opposition. Il n'intervient, en fait, qu'indiqué, appelé, posé, défini par son inverse : le noir visibilise le blanc et lui communique tout son sens. Théologie négative en action (narrative) : ce que tu ne verrais pas t'est manifesté dans ce qui lui est le plus contraire : "Elle était ravissante ainsi, la pauvre petite fille! Les vêtements noirs des deux amis faisaient admirablement ressortir la blancheur de sa robe et l'angélique pureté de son visage; son front surtout, éclairé par la lune, semblait rayonner comme celui d'une créature céleste » (MHP, 1,118).

33 VIII. Le noir ajoute au blanc. La couleur adverse (noire, blanche) figure toujours dans la formule (le portrait, la métaphore) comme le signe supplémentaire dont on se dote pour 
plaire, par élégance vestimentaire ou séduction en général. L'homme "blanc", "distingué ", s'habille de noir, comme Monte-Cristo : "Le comte était vêtu de noir et avec sa simplicité habituelle; son gilet blanc dessinait sa vaste et noble poitrine ; son col noir paraissait d'une fraîcheur singulière, tant il ressortait sur la mate pâleur de son teint ; pour tout bijou, il portait une chaîne de gilet si fine qu'à peine le mince filet d'or tranchait sur le piquet blanc» (CMC, II, 511). A noter que cette «blancheur» du teint vient explicitement du cachot et de la tombe. Donc de l'ombre. Ce que le vulgaire s'empresse d'interpréter comme vampirisme : il suffit de ne pas paraitre manger et d'avoir la carnation idoine pour déporter votre nature du côté du pire. Mais Dumas ne laisse pas son lecteur errer sur ces questions cruciales. Fragilité du blanc donc - c'est une couleur qui ne tient pas; génération spontanée du noir - il advient malgré soi. Cependant, aucun des deux pigments n'est apte à signifier mon intérêt sans l'autre, hors son influence : une femme ne paraît désirable en robe blanche que si l'ânon sur lequel elle est juchée est noir (MHP, II, 36). Intrication valorisante des opposites: le noir signifie la bonne tenue du blanc, tandis qu'inversement le "maure " porte blanc pour se faire reconnaître - c'est du reste sur la combinaison de ces signes que le récit élabore ses déguisements et ses fausses reconnaissances (cf. HMR, 22). Il convient néanmoins d'affiner le tableau, Dumas jouant sur le clavier du noir et du blanc de fort virtuoses compositions. Ainsi, le noir signifie la blancheur du blanc en vertu de sa place: un collier noir autour du cou embellit, certes, celle qui l'a attaché là (et pour cause, il tient sa tête !) (FCV, 356-357) ou apprête la colombe à son rôle de messagère de l'amour (COL, 1) ; inversement, une marque blanche au front - et en forme d'étoile, pour bien assurer le signe - sauve les chevaux noirs de Monte-Cristo et en fait d'incomparables bêtes (CMC, II, 381). Idem pour la "raie blanche" faisant jabot de Black, chien diabolique et providentiel (diabolique en effet, l'animal a le museau «teinté de feu à son extrémité ») (BLA, 1,13-14). Attention à la tâche : elle signifie, au nom de sa couleur, là où elle est, comme elle est, son support. Ce qui est blanc noircit, ce qui est noir blanchit, c'est selon.

IX. Blanc avec noir, noir avec blanc, précarité du mélange, La composition de l'une avec l'autre couleur ne tient pas ; c'est un instable ; cette figure de l'étrange demande d'être levée, jetée ou refusée. Ainsi ne s'étonnera-t-on pas que le «café au lait» soit pour Dumas l'« affreux liquide » par excellence (MHP, I, 128-129). Ou encore, paraît un vieux charbonnier, noirci comme de juste, dont les dents brillent au milieu de la figure : le personnage doit muter, en vertu du principe selon laquelle la pureté se veut, élimine sa tâche, tend à l'homogène. Il sera Dieu venant en aide au héros. Le noir recèle la divinité ; la divinité s'y dissimule et manifeste sa puissance du fond de la nullité qui lui sert d'enveloppe. Pourtant, elle doit (se doit de) surgir, se révéler, dépouiller sa noirceur, «blanchir».

$\mathrm{X}$. Blanc est l'objet du désir ; il est beau d'être blanc ; il est désirable d'être beau. La femme est blanche, par nécessité d'une telle loi $^{23}$. Toutefois, on en sait déjà les raisons, sa "blancheur " ne s'apprécie qu'encadrée, contrastée, mise en valeur par son opposite inférieur et minoritaire : «Amy. - (...) Moi aussi, j'ai mille choses à vous dire, et la première, ma belle vénitienne, c'est qu'au milieu de nos cheveux blonds et de nos yeux bleus, vos cheveux et vos yeux noirs sont toujours ce qu'il y a de plus nouveau et de mieux pour le moment dans nos salons. Eléna. - Si ce n'est, cependant, ce beau cou blanc et ces belles mains blanches, cette taille mince et souple comme une écharpe... Oh ! bien décidément, vous me rangez à l'avis de votre grand poète, et l'Angleterre est un nid de cygnes au milieu d'un vaste étang... » (KEA, 224 (Acte I, scène II - le dialogue 
se joue devant une psyché)). Autre composition narrative du contraste tempéré constitutif de l'attrait féminin : "La jeune fille, quand le prisonnier fit gémir la rampe de l'escalier sous sa main alourdie, entr'ouvrit le guichet d'une chambre qu'elle habitait dans l'épaisseur de cet escalier même. Et la lampe à la main droite, elle éclaira en même temps son charmant visage rose encadré dans d'admirables cheveux blonds à torsades épaisses, tandis que de la gauche elle croisait sur sa poitrine son blanc vêtement de nuit (...). C'était un bien beau tableau à peindre et en tout digne de maitre Rembrandt que cette spirale noire de l'escalier illuminée par le fallot rougeâtre de Gryphus - il s'agit du père de la jeune fille - avec la sombre figure du geôlier au sommet, la mélancolique figure de Cornélius qui se penchait sur la rampe pour regarder, au-dessous de lui, encadré par le guichet lumineux, le suave visage de Rosa, et son geste pudique un peu contrarié peut-être par la position élevée de Cornélius, placé sur ces marches d'où son regard caressait vague et triste les épaules blanches et rondes de la jeune fille » (TUN, 96). La conjonction des caractères du blanc (le corps de Rosa), du noir (l'escalier où la surprend le regard du prisonnier) et du rouge l'émanation du père - je réserve l'analyse de ce troisième élément explique la séductivité fantasmatique de l'apparition féminine. Je me séduis de l'impossible combinaison, contraction, en un corps, sur le corps autre, de ce qui à la fois me pose, me transpose et me nie. "C'était un groupe ravissant, beau, jeune, frais. On eût dit un cygne noir et un cygne blanc se caressant dans un bassin de marbre rose » (MHP, IV , 240). Ravissement énigmatique, au reste, qu'engendre un tel contraste, puisque ce qui s'y dit sous le nom de beauté provient de l'effacement réciproque des signes qui l'établissent : "noir » se résorbe dans l'objet de la contemplation, «blanc» ne subsiste que comme une trace laissée sur la peau, pour notre émerveillement, par suppression, radiation complet de la personne à soi : «Son visage blanc était comme la cicatrice de son deuil: tout son visage, d'une façon égale et lisse, avait un aspect de cicatrice $»^{24}$.

7. Noir et blanc sont en lutte. Tout ce qui précède l'implique: que l'un paraisse, l'autre advient, qui lui barre la route. Attirance des deux principes, répulsion des deux principes, mutualité de l'aversion qu'ils connaissent. Les récits dumasiens sont là pour mettre en scène, expérimenter, ce combat perpétuel. Le bon et le méchant sont à l'œuvre, certes, ni plus ni moins qu'ordinairement en régime romanesque, mais ici les deux principes sont revêtus d'un signe qui les désigne à notre attention : la couleur de la peau. Comme si toute négativité se réfugiait dans les apparences du noir, comme si toute positivité choisissait nécessairement les tonalités de la candeur. Le méchant veut donc dire encore bien autre chose que sa simple méchanceté, nous commençons à nous en rendre compte. Le texte, en tout cas, heurte noir contre blanc; il vit de cette guerre ; il conduit à sa cessation; c'est là et non ailleurs qu'il se fonde. Subsumer (aufheben) ce qui fait opposition et en faire dériver la nature sur ce qui lui est contraire, voilà ce que tente, chez Dumas, une histoire constamment recommencée.

Invincibilité des deux principes, perpétuation de la représentation de leur choc, livre après livre. Insatiabilité de la représentation. Indéfectibilité du nœud que l'histoire doit exposer. J'élabore une lutte - les archives et l'Histoire tout entière, la mémoire des voyageurs et les auteurs des littératures qui me sont accessibles sont le fond où je puise pour cela -, je fais advenir une fin, je mets du sens dans cette fin, je profile dès le début ce qu'elle veut dire, je ferme le livre et recommence : «Et il prit le cadavre de Blanche, l'assit majestueusement sur sa chaise de bois sculpté et lui posa le pied sur la tête sanglante de Mothril, comme les peintres et les sculpteurs ont posé le pied de la Vierge sur la tête brisée du serpent » (BAM, I, 194). Mothril est le maure, Blanche est la reine, 
le metteur en scène est le franc. Mais la mort du premier n'est qu'apparente et le pieux chevalier mettra encore un nombre respectable de pages avant de pouvoir réellement s'en débarrasser. Et encore : l'aventure lui coûtera un bras et c'est ce membre par défaut qui l'entraîne au récit. Etc.

Bien évidemment, la mise en scène de cette campagne sans fin n'est susceptible d'intéresser que sur la base de la contiguïté des deux principes. Noir et blanc sont des éléments «fraternels », connivents, du raisonnement : «Puis la quantité : tout homme est blanc, nul homme n'est blanc, quelque homme n'est pas blanc... Et la relation: le sens doit rester le même pour la relation renversée, puisque la relation par rapport à lui s'établit toujours dans les deux sens à la fois (...). Le sens est toujours double sens, et exclut qu'il y ait un bon sens de la relation ${ }^{25}$. La participation réciproque d'un des deux termes à son adverse nourrit la relation, donc le récit. Ce n'est que parce que le blanc se trouve constamment menacé de dériver et de passer au noir, et parce que celuici, principe instable, désire, pour ainsi dire, de lui-même cesser, que l'histoire trouve à être dite et fait sens. Ainsi en va-t-il pour le combat acharné que se livrent les frères ennemis, prétendants au trône d'Espagne, dans Le Bâtard de Mauléon, Dumas prenant bien soin de les colorer de la bonne façon (BAM, 1,222).

Je procède maintenant, comme annoncé, avec un peu plus de détails, et ouvre trois textes successifs :

\section{Y perdre la peau (dans Le Meneur de loups)}

Le héros, sabotier de son état, mais sabotier envieux des biens et d'un statut social qu'il ne possède pas, fait pacte avec le diable, paru à lui sous l'aspect d'un grand loup noir. Son ange gardien est bien sûr une pure jeune fille qui l'aime, dont le nom dit la blancheur: Agnelette, et la promet aux crocs du fatal adversaire. En contrepartie, Thibault cède au diable, pour chacun des souhaits qu'il forme, l'un de ses cheveux, qui devient roux. Or, malheureusement pour lui, mais providentiellement aussi pour le salut de son âme, l'accomplissement des vœux, mal orientés d'ailleurs, ne comporte pour le héros aucunement la félicité qu'il en attend. Cependant, peu à peu, sa chevelure, de noire qu'elle était, devient flamboyante. Ce pacte sera suivi d'un second, qui doit douer Thibault acculé dans une situation sans issue de l'impunité et de l'immortalité. En échange, il prendra la place du diable et revêtira alors sa livrée noire. Seul un ultime cheveu blanc (ou plutôt crin) témoigne encore de son humanité. Thibault, traqué par une meute acharnée durant la nuit où, annuellement, il doit redevenir mortel, trouve refuge dans un cimetière et précisément dans le tombeau même de sa mère. On y trouvera, au matin, son animale dépouille; il terminera sa vie en repentance dans le couvent voisin. Moralité : on ne change pas de peau; la blancheur est divine; elle ne s'acquiert qu'abandon fait de l'autre peau; c'est par retour à la mère, gage de blancheur, que la blancheur se gagne - après détour et soumission de toute une vie pourtant - au principe diabolique. Le père est absent du texte; le démon noir occupe le devant de la scène, sous la forme animale qui lui est propre : je vois beaucoup de parenté entre cette absence et cette présence.

\section{Sauver sa face (dans Georges)}

41 Ou comment le noir devient blanc sans changer de couleur. Sara, la belle créole blanche, riche héritière de surcroît d'un père planteur sur les terres desquelles travaille toute 
une population d'esclaves, va se baigner - comme il se doit - dans la Rivière noire. Elle revêt pour cela une suggestive tunique blanche et virginale. Jeu d'eau où les témoins que nous sommes la regardent «blanchir » sous l'écume et les jeux de lumière. Survient un affreux requin. Horreur. Un nègre employé dans la propriété du père et amoureux de la jeune fille - comme le Bug-Jargal de Hugo, mais il sauvera, lui, sa maîtresse d'un crocodile - comme on voit, tous les nègres sont sauveurs! - plonge courageusement le poignard à la main. Hélas! le monstre nage plus vite et nous entendons déjà le bruit que font ses dents refermées sur sa proie. Au moment crucial, coups de feu salvateurs : le héros, Georges, métis, riche et civilisé, de retour de Paris pour accomplir une œuvre de justice, intervient: il soustrait la jeune fille au monstre - et au nègre du même coup! - et se la gagne pour lui. Du reste, la conquête de la jeune fille avait déjà commencé lorsque Georges, pour une histoire d'éventail, avait déjà pu lui démontrer sa «blancheur » en parlant la langue... jaune - le chinois - du marchand (GEO, 150, 161, 170-173). Le récit transmue donc de l'identité noire en identité blanche par " efféminisation " (sauvetage et épousailles) et liquidation (par transfert sur plus noir que lui) de la souche esclave. Georges aura encore à mater une révolte d'esclaves pour devenir celui qu'il n'était pas et prendre la place du blanc fiancé légitime : Dumas fait bon poids, on ne blanchit jamais assez son ombre.

\section{Le pur enfantement (dans La Tulipe noire)}

Un homme invente une fleur ${ }^{26}$. A la clef de la découverte : un trésor et une femme blanche et si possible laiteuse - puisque hollandaise - comme il se doit. Cet homme réussit ce miracle de l'inventer noire : Rosa l'aidera pour ce faire. Voici les principaux chaînons du raisonnement narratif :

A. L'origine est blanche; la plante recueille le blanc «dans le germe ». Cependant, cette blancheur est soumise à une - apparemment fatale - noircissure (TUN, 162, 192). Le prodige est donc nécessairement de créer l'impossible teinte, d'atteindre à l'inaccessible essence, à l'hors-nature: "Ce fut vers cette époque que la société tulipière de Harlem proposa un prix pour la découverte, nous n'osons pas dire pour la fabrication de la grande tulipe noire et sans tache, problème non résolu et regardé comme insoluble, si l'on considère qu'à cette époque l'espèce n'existait pas même à l'état de bistre dans la nature » (TUN, 67). Naturaliser le noir, faire du noir une coloration florale, non néfaste donc, telle est la gageure.

B. Le noir s'obtient par lente imprégnation des ténèbres sur le bulbe et la graine; c'est le résultat d'une opération alchimique, dont Dumas prend grand soin de spécifier qu'elle est «innocente » - qu'elle ne doit donc rien au démon - et « enfantine » - c'est le produit d'un « rêve » (TUN, 69).

C. Pureté du caïeu primitif - «aïeul », " aïeux»! - dont le noir tulipier va pouvoir s'épanouir. Le contraste refend la représentation : le caïeu du noir a les propriétés du blanc ! «- Voilà cependant de bien jolis caïeux, dit-il ; comme ils sont lisses, comme ils sont bien faits, comme ils ont cet air mélancolique (sic) qui promet le noir d'ébène à ma tulipe! sur leur peau les veines de circulation ne paraissent même pas à l'œil nu. Oh! certes, pas une tâche ne gâtera la robe de deuil de la fleur qui me devra le jour » (TUN, 77).

D. La pureté miraculeusement conquise de l'impossible couleur - par refoulement et transfert - n'entraîne pourtant pas, pour commencer, la perfection de la fleur 
obtenue: la tulipe noire manque de parfum !... Doter la fleur muette d'une parole spécifique est une tâche surhumaine; ce serait, dit le texte, s'égaler aux plus grands capitaines et dépasser le grand Alexandre lui-même! (cf. TUN, 18). Alexandre rêve Alexandre! Alexandre au secours de l'immaculée Conception!

E. La tulipe, sous la forme inséminatrice du caïeu, est confiée par son créateur et afin de la soustraire aux recherches d'un père tyrannique (il est en prison, son gardien est aussi le père de celle qu'il aime), à une femme, Rosa, la blonde et la blanche, comme la juste. Le héros spécifie bien que celle-ci a pour tâche de protéger ce germe " comme la mère garde son fils » (TUN, 175). La femme recèle et garde ce que l'homme dépose en elle pour épuration. La pureté du fruit, sa propreté, son identité, but du processus, s'obtient donc par mélange (des sexes, des couleurs), dans le couple.

F. Lutte entre le noir, principe mâle d'essence maligne, et le blanc, Rosa, principe féminin, d'inspiration divine. Dans cette lutte, la victoire - c'est-à-dire donc la survie du bulbe miraculeux - suppose un double abandon : Cornelius doit croire sacrifier sa tulipe à celle qu'il aime et celle-ci, réciproquement, faire don de sa "blancheur ». "Jamais l'amour du prisonnier n'avait été aussi réel qu'arrivé au moment où nous sommes parvenus, puisque, nous l'avons dit avec embarras (sic), dans la lutte entre la grande tulipe noire et Rosa, c'était la grande tulipe noire qui avait succombé». Et réciproquement: " Je lui donne (à votre tulipe, dit Rosa) le temps que je donnerais à mon enfant, si j'étais mère. Il n'y a qu'en devenant sa mère (...) que je puis cesser de devenir sa rivale » (TUN, 178-179, 192). Or, cet abandon mutuel des essences est feint : la tulipe ne mourra pas, la pureté ne sera pas perdue (du moins pas avant que le prix n'en ait été versé) ; la substitution ou l'échange des espèces a lieu sans perte. Il le fallait, puisque le noir est le réel (l'« enfant») dont l'abandon ne se peut pas.

G. Pérennité de la marque originaire, qui est blanche. La robe de la tulipe conserve le signe de son appartenance au Bien sous la forme d'un « filet de couleur différente, mince comme un cheveu » (TUN, 205). Aucun noir n'est absolu; le noir ne contient pas en soi son origine ; sa différence s'implique, comme une tache et par un cheveu - qui est la vie.

H. La tulipe-miracle est monnayable ; elle vaut un «trésor »; elle est, du reste, l'objet d'un concours au terme duquel le héros empochera une fortune.

51 Le noir enfanté fleur se conçoit comme une richesse, une propriété, que Cornélius couve "' comme l'avare garde sa première ou sa dernière pièce d'or " (TUN, 175). Le noir vaut le plus; c'est un inimaginable or, une incalculable puissance. Rien peut tout, c'est la revanche.

I. Système christique: on meurt ou faillit mourir pour une "tulipe». Ce "principe de réalité » est un monstre - un «tigre» - par la passion qu'il suscite. C'est le cas du malheureux lord Drammond, très épisodique personnage de Dieu dispose (DDI, VI, 127-128) : son idée le possède - pour la deuxième fois donc, dans l'œuvre dumasienne et conduira à la mort son soupirant de par sa désidérabilité même (DDI, VI, 142, 144). C'est aussi pour sa possession que le richissime aristocrate se fera voleur. En vain (bien sûr!), puisqu'en franchissant le mur du jardin protecteur dans la plus sombre des nuits il se saisira d'une fausse essence : «il s'était trompé de tulipe! Au lieu de la fleur unique, il avait pris une fleur banale, connue dans toutes les serres, et dont il avait lui-même quatre exemplaires. Il poussa un cri » (DDI, VI, 146). Le malheureux anglais entrera en possession, mais trop tard (bien sûr aussi!), de la miraculeuse tulipe, puisque le propriétaire hollandais, par générosité, lui en fera finalement don. Or, conformément à 
l'impulsion dialectique que le texte met en branle, cette fleur signifie tout quoique rien, par simple raison du miracle qu'elle représente : «- En voici une, disait-il, qui surpasse tout ce qu'on peut imaginer de plus merveilleux; le rêve même se confesse vaincu par une réalité aussi désespérante (je souligne); eh bien! ce n'est rien, c'est une fleur insignifiante, c'est un misérable brin d'herbe, à côté de celle que je vais vous montrer (...). Tout à coup son œil tomba sur une tulipe noue, rouge et bleue. Il pâlit et s'élança vers elle (...) - Eh bien! l'avez-vous aussi, celle-là? (dit Tromp (sic), l'imparable propriétaire). - Non, répondit lord Drammond d'une voix étouffée » (DDI, VI, 135, 137-138). Rien plus que rien, et plus désespérant aussi, dans la situation où je suis maintenu d'en être dépourvu : simple fleur, quoique unique. La tulipe tricolore est une femme; elle se désire comme une femme ; il en va de sa propriété, à mort, passionnément, de la même façon (cf. DID, VI, 138, 141). Cet objet - ou plutôt cette figure - du désir provient d'un mixte remarquable, qui redéploie, en une, au sein d'un tableau idéal (« républicain»!), les couleurs françaises, avec inversion de la troisième - le blanc royal ! - substitué par son inverse (cf. DID, VI, 137, 147). D'une fleur comme d'un symbole de soi, dérivé d'une identité nationale jacobine, adorée par un anglais! Le texte ne recule devant aucun luxe ! On voit la chimie et la mystique : le noir se mérite, s'efface aussi, mais s'ennoblit, par la mort qu'il engendre chez qui l'aime. Dieu ne se révèle qu'au pécheur.

J. Le vol du fétiche. La fleur miraculeuse, incarnation de l'impossible rédemption de la noirceur, suscite le désir et provoque le vol. Ce vol démontre sa valeur générale: un symbole engendré pour compenser une perte individuelle - masquée sous la forme de la pauvreté ou du célibat - finit par convenir à tous, dans un récit visiblement acharné à enfouir ses vérités au plus profond: ce qui me constitue se dilue et se dissimule dans l'envie qu'il provoque chez mon voisin. Or, comble du cache et de la dénégation! le voleur de la tulipe noire, qui s'apprête donc à recueillir l'injuste prix de son larcin, prend logement, à Harlem, à l'hôtel du Cygne-Blanc (cf. TUN, 239, 244, 260). Cette surcharge des signes (le blanc sur le noir, l'oiseau sur la fleur) tend à en effacer la vraie valeur : cette méconnaissance est aussi ce qui désespère et le héros et le lecteur. Une substitution des pères, une permutation des signes, le tour est joué, rien ne va plus.

$\mathrm{K}$. Une fille devient mère de ce noir symbolique que lui donne à couver l'hommeauteur. Cette « maternité » la sépare de son propre père. Douleur de celui-ci, horreur de celui-ci, vengeance, menaces. Je lui ouvrirai le cœur, dit-il du donneur, pour voir dedans où il cache son enfant, et, qui plus est, de ce même couteau qui m'a servi à égorger "plus de cinquante coqs noirs» (TUN, 272). Il n'est donc pas facile d'inventer un corps blanc, un corps d'enfant, un corps propre clandestinement au noir : un père veille à la bonne répartition des souches et des races. Le texte de l'impur est parmi nous!

8. Le noir travaille les textes. La charge que recèle ce terme, l'énergie dégagée par cet inadmissible. Redon : «Le noir, est la couleur la plus essentielle ${ }^{27}$, celle aussi que tout conspire à écarter. Comme si un refoulement généralisé, dont les fins n'apparaissent pas, se jouait là. Le noir est le danger de la pensée qu'on a. La part obscure ; la part qu'on ne voit pas, l'incommodante, la revenante. Jarry, par son gros homme: la seule façon, quoique abominable, de se prémunir contre le nègre, de son contact irrévérencieux et contaminateur, est d'en supprimer jusqu'à l'idée ${ }^{28}$. C'est en effet du nègre que cela ressort. Du nègre comme de l'ombre, de sa couleur comme de la nuit, menaçante et suscitatrice. Incarnation de la force négative, agent retors et résistant du mal, il est là, présent dans sa couche, comme ce qui obsède. Rencontre du noir, repoussement $\mathrm{du}$ noir, opiniâtreté de son surgissement, patience infinie des manœuvres qui visent à 
l'écarter. D'un texte ou de textes en lutte contre le noir. Des plaques - des récits - qu'il convient de poser aux endroits (du corps) dont il émane. «La bouche de l'enfer est noire $»^{29}$ : la chose est si bien connue que toute l'affaire est de la clore. Du noir au blanc. De la liquidation de sa nature. De sa sublimation ou de la sublimation des produits bâtards que normalement il engendre. De la pure filiation. De la correction des hérédités manquées et désirées. Car le devenir est réservé au blanc seul : on ne devient bien que blanc, on ne s'identifie que dans cette couleur. Et pis - pis pour un noir qui serait l'auteur de ce conte -, le blanc est le seul bon devenir qui puisse, impossiblement, lui revenir.

De l'œuvre de Dumas comme d'une blanchisserie mentale, où texte à texte on assiste au double jeu d'imprégnation réciproque des couleurs qui font base. A grands coups de symboles, dans le petit jeu fin des opérations narratives. A tel point qu'on dirait que l'histoire - voire l'Histoire, celle des événements humains - suit ce fil : comment blanchir? Comme si le récit n'empruntait ses méandres - et aussi sa verve - qu'à la nécessité de faire arriver d'inadmissibles configurations: j'écris de l'artifice, pour artifice, par artifice.

Prenons Monte-Cristo. Le comte, dont l'identité est tue, que ses ennemis ne reconnaissent pas, que ses amis mêmes ignorent, porte successivement les surnoms nécessaires à ce cache : " major Black », car il s'habille de noir, «lord Ruthwen », car sa figure invraisemblablement pâle l'associe au vampire (cf. CMC, I, 782). Il est, sous ces noms, l'incroyable, le mystère, l'impossible et inflexible agent d'une Providence de fer, devenu par la souffrance ce blanc qu'il n'était pas, ayant accédé au terme d'un long chemin de croix à la seule bonne essence. Tant il est vrai qu'un blanc lui-même a à se blanchir. A plus forte raison si l'on ne l'est pas. Et puis aussi (la dialectique est retorse) puisque les blancs ne sont pas teints de cette blancheur idéale qu'il faut, les pauvres noirs se trouvent du même coup allégés d'une faute qu'ils ne sont pas seuls à porter. Car le blanc bien évidemment n'est pas un fait de race : il se donne à la personne, c'est à la personne aussi qu'on le retire. Comme un signe d'appropriation : le noir manque d'être, le blanc donne l'être. Le teint mental de ma face affirme que je suis ou ne l'affirme pas. Car le blanc se mérite : le récit construit de la couleur - de la nécessité - de l'essence. Toucher au propre, quel délire! Baptêmes nombreux dans l'œuvre de Dumas, où le héros plongé dans toutes les eaux possibles, sous des flux et sous des cataractes, trempé de nuit pour bien faire - c'est le cas du comte précipité dans les eaux nocturnes au bas du château d'If -, la rivière s'appellerait-elle « eau noire » (elle n'en est pas moins incolore! (cf. ALP, 108)), blanchit pour sa peine. Opération lustrale d'un côté, que Dumas emprunte à l'Eglise, épuration symbolique de l'autre qu'il applique à son sujet, à ses transferts, à l'envi et de sa propre autorité. Généalogie et filiation : le texte trame des histoires identificatrices à blancheur forte. Renouer et dénouer, rétablir la bonne essence, par actions concertées sur le père (ou ses substituts et dérivés), rompre d'avec la mauvaise par tout le jeu, contre lui, des combats nécessaires ${ }^{30}$.

Insistance de la démonstration, écrire met du propre, pousse à jouir à l'accession à ce bien-là. Par entremise de l'intrigue ou dans le tout-venant d'un texte apparemment disert et qui noircit le papier pour faire de l'argent - puisque aussi bien c'est ce qu'on dit. En tout cas, exclusion de la chose néfaste, inclusion du teint le plus convenable, passage et transmutation du premier au second au nom du vrai principe : je deviens, je me baptise, je me dépouille de ma peau, je me hisse à l'autre, je me nie et communie sous les deux espèces. 

qu'ils tirent leur origine, n'ont point de mémoire. Le passé leur est aussi inconnu que l'avenir, et ils mourraient plutôt que de dire leur secret ", lit-on dans Les Suppléments au voyage de La Pérouse ${ }^{31}$, c'est une bonne vieille antienne. Or, le nègre Dumas est bavard, lui, et revendique un passé - normal, pour un auteur de romans historiques! - que pour avoir à sa mesure il n'hésite pas à refaire. A refaire, pas à mentir. Le faisant arriver à sa façon et dans le sens qu'il faut aux substituts qu'il imagine lui (même très en dehors de sa concrète apparence). Le noir n'a pas de passé ? Accordons-lui en un dans le temps même où nous pourrons démontrer qu'il s'est défait de sa fausse origine. Tout le problème est là : comment quitter cela qu'on a été sans perte? Les acrobatiques aventures d'Alexandre Dumas le content à satiété. est aussi une remontée aux sources généalogiques - ainsi que la perversion de celles-ci. L'excursion dans les Alpes, qui date de 1832, première d'une longue série d'" Impressions ", conduit Dumas à pied... d'œuvre : au Mont-Blanc. Son récit relate, avec le plus grand luxe, tous les détails de la première ascension (intervenue quarante-six ans plus tôt). Il va à la source et rend visite - afin de garantir la véracité de son texte - à Jacques Balmat, premier à avoir foulé la crête, comme tous les touristes qui l'ont précédé, d'ailleurs. Le miracle est d'abord celui-là : que le Mont-Blanc soit accessible! Dumas met cette affirmation obsessionnelle dans la bouche du guide, mais c'est bien lui qui l'énonce (ALP, 61). Au reste, fait à noter, cette accessibilité est présentée comme le produit du rêve de l'alpiniste. Ascension, naissance: tout indique que cela commute. D'ailleurs, c'est un « docteur » qui accompagne le guide. Ici, creusons et rapportons la scène à celle de l'accouchement de la mère de l'auteur, tel qu'il est rapporté dans les Mémoires. Noir, rouge, bleu sont à la fois les couleurs du bébé qui doit se désentortiller du cordon ombilical et celles du grimpeur arrivant quasi asphyxié sur le dôme : «Je n'étais pas reconnaissable; j'avais les yeux rouges, la figure noire et les lèvres bleues ; chaque fois que je riais ou bâillais, le sang me jaillissait des lèvres et des joues. Enfin, je n'y voyais plus qu'à l'ombre » (ALP, 77). Le fameux Berlick et Jacques sont dans le même cas. Le texte pousse si loin l'analogie qu'il fait boire trois bouteilles au héros de Chamonix pour compenser le cri d'étranglé du malheureux nouveau-né entendu dans la mémoire comme un flacon d'eau de Selz qu'on vide! On voit que ces "couleurs" proviennent au corps, aux orifices, à la figure - là où je parle et comment je le suis. $\mathrm{A}$ cette hauteur - et cela veut dire, dans cette vie - la nature extérieure n'existe plus que sous les deux espèces : noir/blanc, voir/ne pas voir ${ }^{32}$. Noir-bleu- rouge, d'un côté, noir et blanc, de l'autre : la différence est dans le sang qui étouffe. On comprend alors ce que recèlent en vérité les paroles attribuées à l'alpiniste triomphateur : «J'étais au terme de mon voyage. J'étais arrivé là où personne n'était venu encore, pas même l'aigle et le chamois (...) j'étais le roi du Mont-Blanc, j'étais la statue de cet immense piédestal. $\mathrm{Ah} ! »(\mathrm{ALP}, 73)$. De la trajectoire biographique, comme de celle du voyage: on n'a jamais prêté attention au fait que Dumas circule géographiquement en fonction de nécessités tout sauf circonstancielles. C'est ainsi du moins qu'il se représente le monde à parcourir : au guide de Chamonix venu lui rendre visite et auquel il montre le spectacle alors renversant du diorama, c'est le parcours symbolique qui est visible : "Nous arrivions au moment même où la Forêt-Noire disparaissait pour faire place à la vue du MontBlanc; dans le coin du tableau qui commençait à paraître, on distinguait de la neige et des sapins » (ALP, 222). Du fin fond jusqu'au sommet, du noir qui disparaît au blanc qui arrive, tel est le rite, le cycle, la demande et l'écrit. Tous les voyages sont dans l'imaginaire; 
tous les livres sont des voyages, l'essai d'un départ, l'espérance d'un terme. Du signe de la race honnie au signe de la préférée. Dumas se récite pour soi l'inconcevable retournement de l'un dans l'autre, son inverse et son maitre ${ }^{33}$. Question: comment changer la nature d'un signe ? Comme changer d'arbitraire ? Réponse - sans réponse! - : par écrit et projection, les personnages me répondent.

Si le noir est ce dont le propre se dégage - il faut l'aider ! - pour m'être attribué, je ne puis, récursivement, de texte en texte plus avant, que l'exclure, ce blanc, l'inclure, ce noir, dans le geste même qui me fait le poser : en fait, je ne conçois « noir » que « dans l'effroi », comme " asphyxie » - une couleur me noue la gorge! -, « aux deux bouts de la route », je ne conçois blanc qu' " au-dehors", "en haut " et comme absence. Je ne céderai pas à l'empire de l'ombre ; je toucherai à la lumière, pour « aveuglante » qu'elle soit : noir, je me le rends, blanc, je me l'enlève. Le noir s'inaccepte, le noir insiste : je le repousse, tout en le voyant, par fermeture des yeux et report de la vue, tandis que son inverse m'est donné dans un trait de lumière si vif que je me dérobe à lui. Accordé, c'est repris. Repris, c'est remis. Le noir revient dans le regard de qui observe le devenir blanc du temps et de la route.

DUMAS fils : «On ne tire pas de la farine d'un sac à charbon $»^{34}$.

DUMAS père : «Je prends volontiers des bains de lait, en Suisse ; ils sont ici moins chers qu'ailleurs - rien que le prix moyen de deux repas » (ALP, 232).

\section{Ouvrages de Dumas utilisés dans ce travail}

ALP : Les Alpes de. Chamonix à la Grande Chartreuse, Présentation d'Emmanuel FRAISSE, Encre, 1980.

BAM : Le Bâtard de Mauléon (1361-1369), Préface de Gilbert SIGAUX, Genève, Le Cercle du Bibliophile, 1972, 2 vol.

BAL : Black, La Haye, 1858, 3 vol. dessins hors texte par Adrien Marie et de nombreuses vignettes dans le texte, CalmannLévy, s.d. 
TDE : Le Trou de l'enfer, Verviers, Bibliothèque Marabout, 1975.

TUN : La Tulipe noire, Calmann-Lévy, 1925, œuvres complètes d'Alexandre Dumas

\section{NOTES}

1. Cité in A. MAUROIS, Les Trois Dumas, Le Livre de Poche, 1961, p. 344. Ce travail fait partie d'un livre à paraitre aux Presses universitaires de Lille, dans la collection « Objet ».

2. Nodier cité par MAUROIS, op. cit., p. 78. Même jugement sans appel des Goncourt : « Nègre! » (Ibid., p. 395).

3. E. de MIRECOURT, Fabrique de romans, Maison A. Dumas et Cie, 1845, cité par MAUROIS op. cit., p. 205.

4. MAUPERTUIS, Vénus physique, Aubier-Montaigne, 1980, p. 172 : On peut imaginer que «la source des œufs noirs étant épuisée ", l'Ethiopie se peuple irréversiblement d'habitants blancs.

5. Ibid., p. 143. « Peut-être ", ajoute le vieil auteur.

6. L'Homme qui apprenait lentement, Seuil, 1985, p. 174.

7. Cf. Michel SCHNEIDER, Voleurs de mots, Gallimard, 1985, p. 367.

8. Ibid., p. 368.

9. Ecrits sur le livre. Choix de textes. Précédé par Henri MESCHONNIC, Mallarmé au-delà du silence, L'Éclat, 1985, p. 139.

10. Jean RISTAT, Qui sont les contemporains, Gallimard, 1975, p. 296.

11. MALLARMÉ, op. cit., p. 126.

12. Ibid, p. 146.

13. Jacqueline KELEN, La Tache. Opuscule furtif sur les salissures du monde, Scarabée, 1984, p. 48.

14. Cité in Art Press, 70, mai 1983, p. 32.

15. Du reste, la traite s'est poursuivie avec et sans justification jusque tard dans le XIX ${ }^{\mathrm{e}}$ siècle, en 1862, lorsque Lincoln décida de signer un traité autorisant l'Angleterre à fouiller les navires suspects battant pavillon américain.

16. En 1806, Alexandre n'a pas quatre ans...

17. Ne dit-on pas « rose » pour « noir » dans le créole de l'Ile Maurice?

18. Le sigle renvoie à la bibliographie en fin d'article.

19. Je renvoie, ici à Ch. G., Le Fond du texte. Alexandre Dumas. Berlick/ Berlock, in Rapports, 3,105-112 ; 4,137-148.

20. Coupable indirect de la mort du général et responsable de la ruine maternelle. Au reste, la position de l'écrivain envers lui ne manque pas d'être ambiguë - je m'en explique ailleurs.

21. Cité in 'Claude SCHOPP, Alexandre Dumas. Le Génie de la vie, Mazarine, 1985, p. 470.

22. (21 bis) Cependant, la "pâleur » non tempérée, non naturelle, fait mystère et implique la menace.

23. "Blanche, très blanche ", c'est le nom et la couleur, multiple au reste, et profonde, de la très sainte Veuve noire, selon Ramon GOMEZ DE LA SERNA, dans l'un de ses inimitables livres (La Veuve blanche et noire, Le Sagittaire, 1924).

24. Ibid.,yp. 231-232.

25. DELEUZE, Logique du sens, UGE-10/18, 1973, p. 47. (Je détruis le contexte).

26. «La passion des fleurs est une des plus naturelles au cœur de l'homme » (DDI, VI, 129). « C'est offenser Dieu que de mépriser les fleurs » (TUN, 61). 
27. A Moi-même, Corti, 1961, p. 124.

28. Oeuvres complètes, Gallimard-Bibliothèque de la Pléiade, 1972, I, p. 608.

29. (27bis) H. GERBEAU, Les Esclaves noirs. Pour une histoire du silence, Balland, 1970, p. 13.

30. «Cecco eut l'instinct d'être Noir, peut-être parce que son père était Blanc». En d'autres temps, en d'autres lieux, les luttes se ressemblent (cf. M. SCHWOB, «Vies Imaginaires », in Le Roi au masque d'or, UGE-10/18,1979, p. 223).

31. De Yann GAILLARD, Essai sur les voyages imaginaires et autres au 18e siècle, Papyrus/Nadeau, 1980, p. 186, J'ai consacré une discussion à ce passage, cf. note 19.

32. Cf. le témoignage de la première femme « hissée » au Mont-Blanc (ALP, 255).

33. FORNERET, lui, choisit la voie inverse : noircir, et couvre ses textes, pour les désaffecter, d'une impossible signature : Sans titre, Encore un an de sans titre, PAR UN HOMME NOIR BLANC DE VISAGE..

34. Cité in Maurois, op. cit., p. 462.

\section{AUTHOR}

\section{CHARLES GRIVEL}

Université de Mannheim (R.F.A.) 